\title{
A NOTE ON AN INEQUALITY WITH NON-CONJUGATE PARAMETERS
}

\author{
by P. L. WALKER
}

(Received 19th May 1972, revised 8th November 1972)

The inequality

$$
\int_{0}^{\infty} \int_{0}^{\infty} \frac{f(x) g(y)}{x+y} d y d x<\pi \operatorname{cosec}(\pi / p)\|f\|_{p}\|g\|_{q},
$$

which is valid for positive, non-null $f, g$ in the spaces $L^{p}(0, \infty), L^{q}(0, \infty)$, where $p>1,(1 / p)+(1 / q)=1$, is a well-known generalisation of the classical inequality of Hilbert (see for instance Chapter 9 of Hardy, Littlewood, and Polya (1)).

It is also shown in (1) that the constant $\pi \operatorname{cosec}(\pi / p)$ is best possible.

The case when $p, q$ are not conjugate parameters, but are restricted only by $p>1, q>1,(1 / p)+(1 / q) \geqq 1$ is also considered in (1). If $p^{\prime}=p /(p-1)$, $q^{\prime}=q /(q-1)$ are the conjugate indices to $p, q$, and we write

$$
\lambda=\left(1 / p^{\prime}\right)+\left(1 / q^{\prime}\right)=2-(1 / p)-(1 / q) \text {, then } \lambda \in(0,1],
$$

and the inequality takes the form

$$
\int_{0}^{\infty} \int_{0}^{\infty} \frac{f(x) g(y)}{(x+y)^{\lambda}} d y d x<K(p, q)\|f\|_{p}\|g\|_{q} .
$$

The upper estimate

$$
K(p, q) \leqq\left\{\pi \operatorname{cosec}\left(\pi / \lambda p^{\prime}\right)\right\}^{\lambda}
$$

was first obtained by Levin (2) and later, more elegantly by Bonsall (3). Evidently this value agrees with that in the conjugate case when $\lambda=1$.

The object of the present note is to show that although the problem of whether (ii) gives the best possible of $K(p, q)$ in all cases is still apparently open, the estimate is asymptotically best possible, in the sense that if say $q$ is fixed then $K(p, q)\left\{\pi \operatorname{cosec}\left(\pi / \lambda p^{\prime}\right)\right\}^{-\lambda} \rightarrow 1$ as $p \rightarrow 1$ (and so $p^{\prime} \rightarrow \infty$ ).

In order to obtain lower estimates for $K(p, q)$, we take the following functions for $f, g$ :

$$
\{f(x)\}^{p}=\left\{\begin{array}{lr}
x^{-1-a} & (x \geqq 1), \\
0 & (0 \leqq x<1),
\end{array} \quad\{g(y)\}^{q}= \begin{cases}y^{-1-b} & (y \geqq 1), \\
0 & (0 \leqq y<1) .\end{cases}\right.
$$

In this case $\|f\|_{p}=a^{-r},\|g\|=b^{-s}$, where we have written $r, s$ for $(1 / p)$, $(1 / q)$ respectively. Then

$$
\begin{array}{r}
\int_{0}^{\infty} \int_{0}^{\infty}(x+y)^{-\lambda} f(x) g(y) d x d y=\int_{1}^{\infty} x^{-r(1+a)} \int_{1}^{\infty}(x+y)^{-\lambda} y^{-s(1+b)} d y d x \\
=\int_{0}^{\infty} x^{-1-a r-b s} \int_{1 / x}^{\infty}(1+t)^{-\lambda} t^{-s(1+b)} d t d x
\end{array}
$$

where we have written $y=x t$. 
We now interchange the order of integration to obtain

$$
\begin{aligned}
(a r+b s)^{-1}\left[\int_{0}^{1}(1+t)^{-\lambda} t^{a r-s} d t+\int_{1}^{\infty}(1+t)^{-\lambda} t^{-s(1+b)} d t\right] \\
=(a r+b s)^{-1} \int_{0}^{\frac{1}{2}}\left\{u^{a r-s}(1-u)^{-r(1+a)}+u^{b s-r}(1-u)^{-s(1+b)}\right\} d u
\end{aligned}
$$

where the substitutions $t=u(1+t)$, and $1=u(1+t)$ have been used.

It follows that

$$
K(p, q)>a^{r} b^{s}(a r+b s)^{-1} \int_{0}^{\frac{1}{2}}\left\{u^{a r-s}+u^{b s-r}\right\} d u
$$

where the negative powers of $(1-u)$ have been suppressed.

Hence

$$
K(p, q)>a^{r} b^{s}(a r+b s)^{-1}\left[\left(a r+s^{\prime}\right)^{-1} 2^{-\left(a r+s^{\prime}\right)}+\left(b s+r^{\prime}\right)^{-1} 2^{-\left(b s+r^{\prime}\right)}\right],
$$

where $s^{\prime}=1-s=1 / q^{\prime}, r^{\prime}=1-r=1 / \mathrm{p}^{\prime}$.

In order to obtain an asymptotic estimate as $p^{\prime} \rightarrow \infty$, we allow $b$ to tend to zero as $c / p^{\prime}$ for constant $c$. Elementary calculus shows that the most favourable value of $c$ is given by $c=q^{\prime}$; hence we take $c=q^{\prime}, b=q^{\prime} / p^{\prime}$, and symmetrically, $a=p^{\prime} / q^{\prime}$. In this case $b s+r^{\prime}=q^{\prime} / p^{\prime}$ also, and we obtain from the second term of the above estimate for $K(p, q)$, that

It follows that

$$
K(p, q)>\left(q^{\prime} / p^{\prime}\right)^{s-r-1}(a r+b s)^{-1} 2^{-q^{\prime} r^{\prime}} .
$$

$K(p, q)\left\{\pi \operatorname{cosec}\left(\pi / \lambda p^{\prime}\right)\right\}^{-\lambda}>$

$\left\{\left(p^{\prime} / q^{\prime}\right)^{r}\left(p^{\prime} / q^{\prime}+q^{\prime} / p^{\prime}-\lambda\right)^{-1}\right\} \cdot\left\{p^{\prime} \sin \left(\pi / \lambda p^{\prime}\right) / q^{\prime} \pi\right\}^{s^{\prime}} .\left\{\sin \left(\pi / \lambda p^{\prime}\right) 2^{-q^{\prime}} \pi^{-1}\right\}^{r^{\prime}}$. As $p \rightarrow 1$, we have $p^{\prime} \rightarrow \infty, r^{\prime} \rightarrow 0, \lambda \rightarrow 1 / q^{\prime}$, and it is easily seen that each bracketed term tends to unity.

\section{REFERENCES}

(1) Hardy, Littlewood and Polya, Inequalities (Cambridge, 1934).

(2) V. Levin, On the two-parameter extension and analogue of Hilbert's inequality, J. London Math. Soc. 11 (1936), 119-124.

(3) F. F. Bonsall, Inequalities with non-conjugate parameters, Quart. J. Math. Oxford Ser. (2) 2 (1951), 135-150.

\section{UNIVERSITY OF LANCASTER}

